\title{
Dream Generation and Recall in Daytime NREM Sleep of Patients With Narcolepsy Type 1
}

\author{
Carlo Cipolli' ${ }^{1}$ Fabio Pizza ${ }^{2,3}$, Claudia Bellucci ${ }^{1}$, Michela Mazzetti ${ }^{1}$, Giovanni Tuozzi ${ }^{4}$, \\ Stefano Vandi, ${ }^{2,3}$ and Giuseppe Plazzi ${ }^{2,5 *}$
}

\begin{abstract}
${ }^{1}$ Department of Specialty, Diagnostic and Experimental Medicine, University of Bologna, Bologna, Italy, ${ }^{2}$ Department of Biomedical and Neuromotor Sciences, University of Bologna, Bologna, Italy, ${ }^{3}$ stituto di Ricovero e Cura a Carattere Scientifico "Istituto delle Scienze Neurologiche" di Bologna, Bologna, Italy, ${ }^{4}$ Department of Psychology, University of Bologna, Bologna, Italy, ${ }^{5}$ Department of Biomedical, Metabolic and Neural Sciences, University of Modena and Reggio Emilia, Modena, Italy
\end{abstract}

\section{OPEN ACCESS}

Edited by:

Wei Wang,

Norwegian University of Science and Technology, Norway

Reviewed by: Mark Blagrove, Swansea University, United Kingdom Michelle Carr, Swansea University, United Kingdom

*Correspondence: Giuseppe Plazzi giuseppe.plazzi@unibo.it

Specialty section: This article was submitted to Sleep and Circadian Rhythms, a section of the journal

Frontiers in Neuroscience

Received: 21 September 2020 Accepted: 28 October 2020 Published: 27 November 2020

Citation:

Cipolli C, Pizza F, Bellucci C, Mazzetti M, Tuozzi G, Vandi S and Plazzi G (2020) Dream Generation and Recall in Daytime NREM Sleep of Patients With Narcolepsy Type 1.

Front. Neurosci. 14:608757. doi: 10.3389/fnins.2020.608757
The less rigid architecture of sleep in patients with narcolepsy type 1 (NT1) compared with healthy subjects may provide new insights into some unresolved issues of dream experience (DE), under the assumption that their DE frequencies are comparable. The multiple transition from wakefulness to REM sleep (sleep onset REM period: SOREMP) during the five trials of the Multiple Sleep Latency Test (MSLT) appears of particular interest. In MSLT studies, NT1 patients reported a DE after about $80 \%$ of SOREMP naps (as often as after nighttime REM sleep of themselves and healthy subjects), but only after about $30 \%$ of NREM naps compared to $60 \%$ of daytime and nighttime NREM sleep of healthy subjects. To estimate accurately the "real" DE frequency, we asked participants to report DE ("dream") after each MSLT nap and, in case of failure, to specify if they were unable to retrieve any content ("white dream") or DE did not occur ("no-dream"). The proportions of dreams, white dreams, and no dreams and the indicators of structural organization of DEs reported after NREM naps by 17 adult NT1 patients were compared with those reported by 25 subjects with subjective complaints of excessive daytime sleepiness (sc-EDS), who take multiple daytime NREM naps. Findings were consistent with the hypothesis of a failure in recall after awakening rather than in generation during sleep: white dreams were more frequent in NT1 patients than in sc-EDS subjects (42.86 vs $17.64 \%$ ), while their frequency of dreams plus white dreams were similar (67.86 and $61.78 \%)$ and comparable with that of NREM-DEs in healthy subjects. The longer and more complex NREM-DEs of NT1 patients compared with sc-EDS subjects suggest that the difficulty in DE reporting depends on their negative attitude toward recall of contents less vivid and bizarre than those they usually retrieve after daytime SOREMP and nighttime REM sleep. As this attitude may be reversed by some recall training before MSLT, collecting wider amounts of DE reports after NREM naps would cast light on both the across-stage continuity in the functioning of cognitive processes underlying DE and the difference in content and structural organization of SOREM-DEs preceded by N1 or also N2 sleep.

Keywords: dream experience, narcolepsy type 1, multiple sleep latency test, SOREMP sleep, NREM sleep, dream recall 


\section{INTRODUCTION}

Dream experience (DE) of patients with chronically altered sleep organization may provide important insights into how neurophysiological and psychological processes interact in its generation (for review, see Schredl, 2009). DE of patients with narcolepsy type 1 (NT1, i.e., with cataplexy), which is pathophysiologically linked to the loss of the hypothalamic neurons producing hypocretin (American Academy of Sleep Medicine $[\mathrm{AASM}], 2014)$, is of potentially high theoretical interest, notwithstanding the low prevalence rate of this brain disease (ranging from 25 to 50 out of 100,000 people: Bassetti et al., 2019). Indeed, its hallmarks are sleep fragmentation, several dissociated REM-sleep/wake events (with intrusion of cataplexy, sleep-related paralyzes, and hallucinations into wake and of lucidity and enactment into REM sleep), and diurnal hypersomnolence. The latter often leads to an untimely fast transition (in less than $15 \mathrm{~min}$ ) from wakefulness to REM sleep (sleep onset REM period: SOREMP) at nighttime (Rechtschaffen et al., 1963a), and daytime sleep (Dement et al., 1966).

The occurrence of two or more SOREMPs during the Multiple Sleep Latency Test (MSLT), which remains the most specific neurophysiological marker of NT1 disease (Richardson et al., 1978; Carskadon et al., 1986), seems of particular interest, under the assumption that DE frequencies of NT1 patients are comparable to those of healthy subjects. MSLT studies, which provide five opportunities (i.e., trials) to quantify sleep propensity and detect SOREMPs (in about two thirds of naps: Drakatos et al., 2013a,b), have already shown that DEs reported by NT1 patients after SOREMP naps are comparable in frequency (about $80 \%$ : Benbadis et al., 1995; Waihrich et al., 2006; Cipolli et al., 2020) and structural organization with DEs they report after awakening from nighttime REM sleep (Cipolli et al., 2020). The latter DEs in turn have shown to be similar to those reported by healthy subjects after late-night REM sleep (Cipolli et al., 2008; Mazzetti et al., 2010). Moreover, episodes of lucid dreaming (Fosse, 2000; Dodet et al., 2015; Rak et al., 2015) and of dream enactment (Bellucci et al., 2016) occur also in DEs of daytime SOREMP naps.

On the contrary, the available estimates of the frequency of NREM-DEs are discrepant. On the one hand, DE was reported by NT1 patients after MSLT naps with NREM sleep (hereinafter NREM naps) much less frequently (about 30\%: Benbadis et al., 1995; Waihrich et al., 2006; Schinkelshoek et al., 2018) compared with not only their SOREMP naps but also nighttime and daytime NREM sleep of healthy subjects (about 60\%: for review, see Nielsen, 2000, 2011b). On the other hand, in an experimental study NT1 patients reported DE with a similarly high frequency after one or two around-noon naps with NREM (about 80\%) or SOREM sleep (90\%) (Vogel, 1976). This discrepancy suggests that DE may be generated during daytime NREM sleep more frequently than usually reported.

To ascertain the "real" frequency of NREM-DE, here we asked NT1 patients not only to report DE (so-called "dream") after each MSLT nap, but also, in the case of failure, to specify if they were simply unable to retrieve any content ("white dream") or believed that no DE occurred ("no-dream"). Then, the proportions of dreams, white dreams, and no-dreams and the indicators of structural organization of DEs reported by NT1 patients after NREM naps were compared with those of DEs of individuals with subjective complaint of excessive daytime sleepiness (scEDS). These subjects were chosen as controls because they can take multiple daytime naps (differently of healthy subjects: Carskadon et al., 1986) without (or rarely) reaching SOREMP sleep (differently of NT1 patients: American Academy of Sleep Medicine [AASM], 2014). In this way, we attempted to estimate both the "real" frequency of DE during NREM naps and the difficulty of recalling NREM-DE contents for NT1 patients, in keeping with the general presupposition that the more complex and the longer the $\mathrm{DE}$, the easier the retrieval of its contents (Foulkes and Schmidt, 1983).

\section{MATERIALS AND METHODS}

\section{Participants}

Within the framework of a wide research program on the frequency, content, and structural characteristics of DEs in NT1 patients, we considered for the present study both the DEs of patients with final diagnosis of NT1 who had one or more MSLT naps with only NREM sleep and the DEs of sc-EDS subjects, who have almost all NREM naps.

Both NT1 patients and sc-EDS subjects were retrospectively selected among those consecutively recruited during the diagnostic procedure for suspected narcolepsy at the Narcolepsy Center of the University of Bologna from June 2018 to May 2020. They were drug free (i.e., drug naïve or after a 3 weeks discontinuation) at diagnostic workup that included the following procedures (Pizza et al., 2013): (i) clinical evaluation; (ii) subjective sleepiness assessment (Epworth Sleepiness Scale, ESS) (Vignatelli et al., 2003); (iii) $48 \mathrm{~h}$ continuous polysomnographic (PSG) recording (24 h for adaptation and $24 \mathrm{~h}$ for diagnostic purposes); (iv) five naps MSLT (Littner et al., 2005); (v) in-laboratory test to elicit cataplexy (Pizza et al., 2013; Vandi et al., 2019); and (vi) blood test and, whenever possible, lumbar puncture to search for the human leukocyte antigen (HLA) DQB1*0602 allele (Mignot et al., 2006) and cerebrospinal hypocretin-1 levels, respectively. According to the current international criteria (American Academy of Sleep Medicine [AASM], 2014), a final diagnosis of NT1, narcolepsy without cataplexy (narcolepsy type 2: NT2), idiopathic hypersomnia (IH), or sc-EDS was provided.

To be eligible for the study, participants had also to have fulfilled the following criteria: (i) age between 18 and 50 years; (ii) 8 or more years of education; (iii) no history of neurological, psychiatric, or sleep comorbidity; (iv) ability to recall at least one dream per week (retrospectively evaluated over the previous 2 months); and (v) lack of global and memory-specific cognitive deficits, i.e., without scores below the cutoff points of mild deficit at Wechsler Adult Intelligence Scale-Revised (WAIS-R, Wechsler, 1981) for global and specific cognitive functions, and Wechsler Memory Scale (WMS, Wechsler, 1987) for short- and long-term memory. The 67 eligible participants were requested to report the DE developed during each MSLT nap and signed 
their written informed consent according to the study protocol approved by the local Ethics Committee.

\section{Procedure}

Before the first MSLT trial, participants were instructed that after each MSLT trial they would be asked by an investigator (C.B., blind to participants' clinical diagnosis) to provide a report of the mental experience developed during sleep (using the classical Foiulkes (1962) instruction, "Would you tell me whatever was going through your mind before awakening?"). According to Cohen's (1972) criteria, participants could be a) able to report contents of the previous mental experience ("dream"), b) unable to recall any content of the mental experience felt ("white dream"), or c) unable unable to recall any experience before awakening ("no dream"). In the first case, after the free (i.e., spontaneous) report, they would be asked again: "Could you remember one or more further events of the same mental experience you have just reported?" and thus could further complete their report.

\section{Sleep Scoring and Analysis of Dream Reports}

PSG recordings of all participants were scored by a boardcertified PSG technician (S.V.) and a blind expert neurologist (L.V.) according to the international criteria (Iber et al., 2007). At the end of this analysis, 5 patients showing a comorbid sleep disorder at $48 \mathrm{~h}$ continuous PSG were excluded from the study.

The following sleep parameters were calculated for MSLT naps Time in bed (TBT), Total sleep time (TST), sleep latency (SL), wakefulness after sleep onset (WASO), sleep efficiency (SE), duration of each sleep stage (non-REM sleep stage 1, 2, and 3, i.e., N1, N2, N3), and occurrence of SOREMP sleep (whose duration was not considered for the study). Hypnograms of SOREMP and NREM naps are exemplified in Figure 1.

Sleep recordings and reports of NT1 patients with one or more NREM naps (17 out of 37) and of all sc-EDS subjects $(n=25)$ were considered for statistical analyses.

\section{Dream Recall Frequency}

The proportions of dreams, white dreams, and no dreams were calculated separately out of the number of naps. The proportion of dreams informs us about the perceptual and emotional characteristics and the structural organization of NREM-DE, while the sum of the proportions of dreams and white dreams allows estimating the "real" production (as self-estimated occurrences) of DE during sleep (Fazekas et al., 2019). Indeed, the main neural EEG correlates of the sleep periods preceding dreams and white dreams are very similar, unlike those preceding no dream, for NREM sleep as well as REM sleep in healthy subjects (Siclari et al., 2017). By comparing the two measures, we attempted also to account for the previous discrepant NREMDE estimates obtained in MSLT studies compared to the Vogel's (1976) experimental study.

\section{Report Analysis}

The verbatim transcripts of the whole (i.e., spontaneous plus possibly prompted) DE reports were pruned from clauses not related to or repetitive of dream contents and comments and then were scored by two expert psycholinguists (M.B. and E.R.), unaware of the study aims, of the diagnosis and type of SOREMP/NREM naps. They had to identify independently the structural organization of DEs using the rules of a story grammar (Mandler and Johnson, 1977), which allow identifying large semantic units, which are conceptual in nature, and their causal and temporal relationships (for a detailed description, see Cipolli and Poli, 1992).

The outcome of story-grammar analysis is a tree structure going from the top constituent (Story, namely one or more events linked by the same Setting and Characters) to the basic nodes (Statements, describing either a State or Event). In its simplest form, a (dream-)story consists of a Setting (time and place of the event to be narrated) and an Event structure, with one or more Episodes, each having several intermediate constituents (for an example, see Figure 2).

Inter-scorer agreement was higher than $96 \%$ in parsing statements and classifying statements into intermediate constituents, $95 \%$ in classifying constituents into episodes, and complete $(100 \%)$ in classifying episodes into dream-stories. The few cases of disagreement were solved through discussion between the two psycholinguists. The values of interscorer agreement were high because the story-grammar rules were explicit and not ambiguous.

The following indicators were calculated: (a) the report length (i.e., number of statements per report), (b) the number of dream-stories (i.e., sequences of connected events with the same characters and setting) per report, (c) the length of dream-stories (measured as number of statements), and (d) the indicators of structural organization of dream-stories (Cipolli et al., 1998), namely:

(d1) Context organization (i.e., number of statements per dream-story describing Setting), which is indicative of recall accuracy;

(d2) Sequential (i.e., temporal) Organization (i.e., number of statements per story realizing the actions of Event structure), which is indicative of coherence of dream contents;

(d3) Hierarchical organization (i.e., number of episodes per story), which is indicative of planning of dream plot.

\section{Data Analysis}

SPSS version 21 was used for the analysis of parametric (ANOVA) and non-parametric data $\left(\chi^{2}\right)$. All statistical tests were two-tailed, and alpha level was fixed at 0.05 .

\section{RESULTS}

\section{Demographic, Psychometric, and PSG Data of NT1 Patients and sc-EDS Subjects}

NT1 patients with NREM naps and sc-EDS subjects did not differ significantly for gender ( 7 male and 10 female NT1 patients vs 16 male and 9 female sc-EDS subjects: $\chi^{2}=2.129$, n.s.), as well 

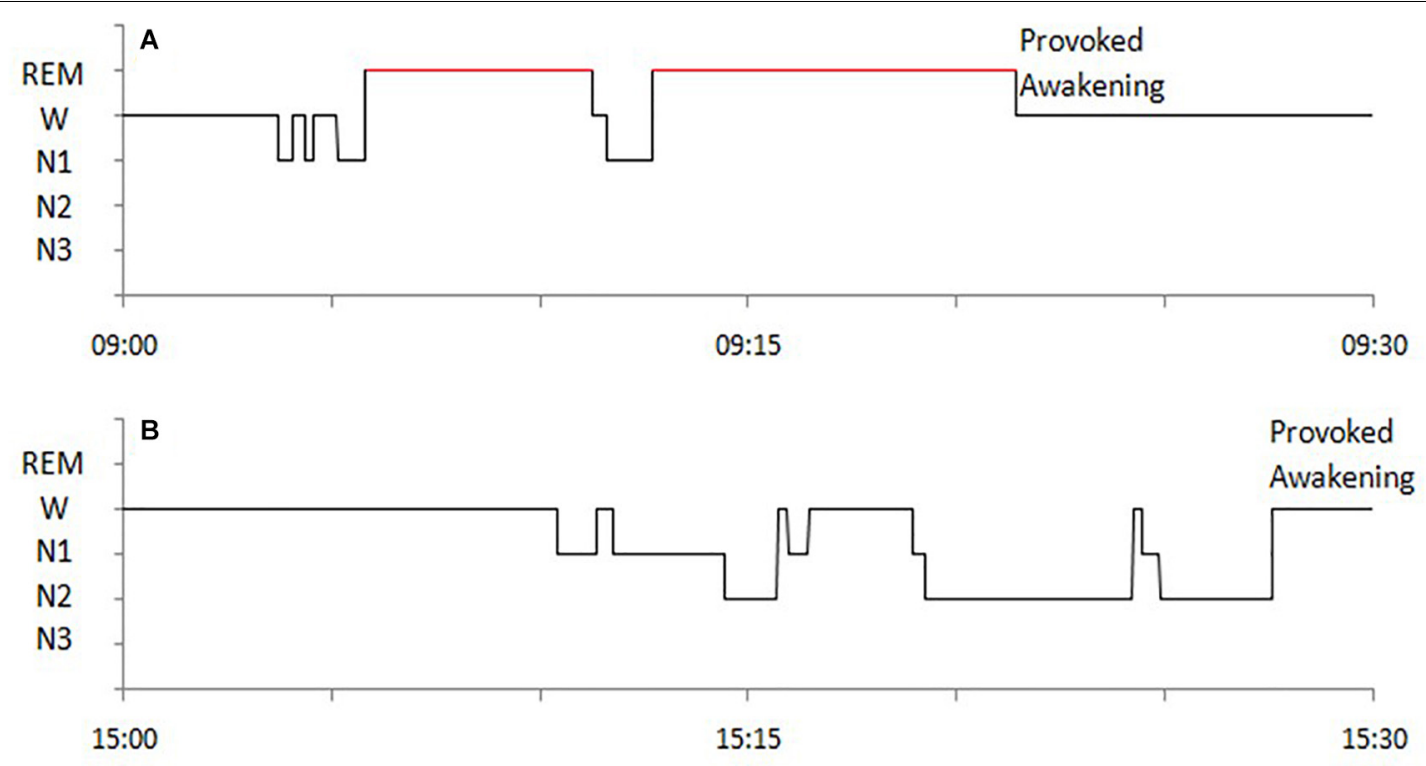

FIGURE 1 | Examples of hypnograms of MSLT naps with SOREMP sleep (A) and with NREM sleep (B) of a NT1 patient.

as for age and years of education (see Table 1a) and values of psychometric indicators (see Table $\mathbf{1 b}$ ).

Table 2 presents the occurrences of naps at MSLT trials, which were more frequent in NT1 patients than sc-EDS subjects $(97.65$ vs $\left.86.40 \%: \chi^{2}=18.776, p<0.001\right)$. The proportion of SOREMP naps was obviously much higher in NT1 patients compared with sc-EDS subjects, in whom it was negligible (66.27 vs $4.63 \%$ : $\left.\chi^{2}=82.979, p<0.001\right)$.

The values of sleep parameters of NREM naps of NT1 patients were significantly higher for sleep efficiency and duration of N2 sleep and lower for TIB, sleep latency, and WASO compared with those of sc-EDS subjects (see Table 1c).

\section{Frequency of Dream Recall}

In NT1 patients, the proportion of DE reports (i.e., dreams) was significantly lower for NREM naps than for SOREMP naps (25 vs $63.64 \%$ : $\left.\chi^{2}=16.049, p<0.001\right)$, while the proportion of white dreams was significantly higher (42.86 vs $20 \%: \chi^{2}=4.836$, $p<0.05$ ) (see Table 2).

The sum of the proportions of dreams and white dreams was significantly lower in NREM naps than in SOREMP naps (67.86 vs $83.64 \%$ : $\left.\chi^{2}=6.166, p<0.025\right)$, while it was similar to that in NREM naps of sc-EDS subjects $\left(61.17 \%\right.$ : $\chi^{2}=0.442$, n.s.). However, the proportions of NREM naps with dreams or white dreams differed significantly in the two groups of participants $\left(\chi^{2}=6.478, p<0.02\right)$, the former being lower $(25.00$ vs $43.69 \%)$ and the latter being higher (42.86 vs $17.48 \%)$ in NT1 patients compared with sc-EDS subjects (see Table 2).

Table 3 presents the occurrences of dreams, white dreams, and no dreams in NREM naps of NT1 patients and sc-EDS subjects according to the sleep stage at the end of MSLT trials.

In NT1 patients, the end of NREM naps was more frequent in N2 sleep (64.29 vs 39.81\%: $\left.\chi^{2}=5.294, p<0.025\right)$ and less frequent in wakefulness compared with sc-EDS subjects (7.14 vs 31.07\%: $\left.\chi^{2}=7.924, p<0.01\right)$.

Table 4 presents the values of length and structural organization in SOREMP-DEs and NREM-DEs of NT1 patients and in NREM-DEs of sc-EDS subjects and the results of the oneway ANOVAs.

The comparison of DEs reported by 6 NT1 patients after both SOREMP and NREM naps showed that reports and dreamstories were significantly shorter after NREM sleep, while the number of dream-stories did not significantly differ. Moreover, dream-stories of NREM naps showed a lower sequential (significantly) and hierarchical organization (by trend) compared with dream-stories of SOREMP naps, while the contextual organization did not differ significantly.

On the contrary, NREM-DEs of 7 NT1 patients showed that both reports (by trend) and dream-stories (significantly) were longer than those of 16 sc-EDS subjects, while the number of dream-stories did not differ significantly. Moreover, dreamstories of NT1 patients showed a significantly higher contextual and sequential (but not hierarchical) organization compared with those of sc-EDS subjects.

\section{DISCUSSION}

The present study attempted for the first time to estimate both the "real" frequency of DE generated during MSLT naps with NREM sleep of NT1 patients by collecting not only their DE reports (dreams) but also self-evaluations of DE occurrence when they were not able to retrieve any content (white dreams), and their difficulty to retrieve contents of NREM-DE. To this aim, their frequency of dreams and white dreams and the values of the indicators of structural organization of DE reports (i.e., dreams) were compared with those of sc-EDS subjects (who likewise can 


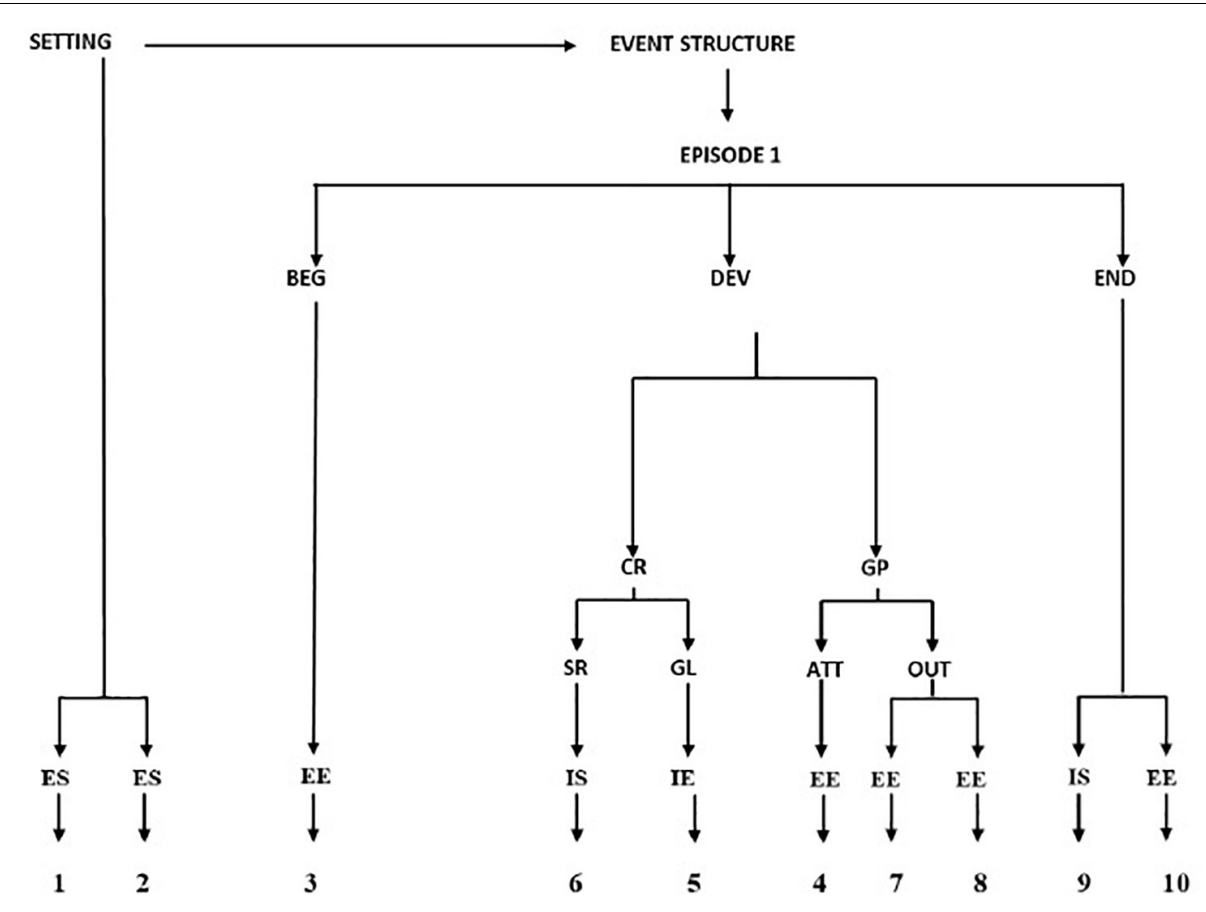

\section{Statements}

ES S1 I was with my mother in a room

ES S2 The environment was similar to this, but there was an armchair instead of a bed

EE S3 A guinea pig was stuck in the closet door

EE S4 (then) I got up from the armchair

IE S5 (because) I wanted to free it

IS S6 I was very anxious

EE S7 (because) I could not free it

EE S8 (finally) I took it and released it

IS S9 (then) I was very happy

EE S10 (and also) My mother heaved a sigh of relief

Legends: $\mathrm{BEG}=$ Beginning; $\mathrm{DEV}=$ Development $\mathrm{END}=$ Ending; $\mathrm{CR}=$ Complex Reaction; $\mathrm{GP}$

$=$ Goal Path; $\mathrm{SR}=$ Simple Reaction; GL = Goal; ATT = Attempt; OUT = Outcome; ES = External

State; $\mathrm{EE}=$ External Event; IE = Internal Event; IS = Internal State.

FIGURE 2 | Dygraph and Statements of a dream experience reported by a NT1 patient after a nap with NREM sleep.

take multiple NREM naps over the day: American Academy of Sleep Medicine [AASM], 2014).

Before discussing the findings obtained, it seems worth pointing out that although NREM naps occurred only in 17 out of 37 NT1 patients examined, their main neurophysiological and psychological characteristics appear reliable. Indeed, the proportions of NREM and SOREMP naps in MSLT of these 17 NT1 patients (28 and 55 out of 83 trials with naps, i.e., 33.74 and $66.26 \%$, respectively) were consistent with those reported in the literature (Drakatos et al., 2013a,b). Moreover, the frequency of NREM naps with DE report (25\%) was similar to those obtained in previous MSLT studies (about 35\%, Benbadis et al., 1995; 30\%, Waihrich et al., 2006; 28\%, Schinkelshoek et al., 2018). Therefore, with the caution required by the small size of our sample, it can be argued that the results of the comparison of data of NT1 patients with those of sc-EDS subjects provide reliable indications on NREM-DE generation and recall. These indications are obviously more cogent for DEs generated during N2 sleep, given the longer duration of this stage and the higher proportion of NREM naps ending with it in NT1 patients compared with sc-EDS subjects.

\section{PSG Data}

The analysis of PSG recordings showed that the sleep propensity, as expression of the homeostastic pressure, was greater and more homogeneous over the trials of NT1 patients compared with those of sc-EDS subjects, in keeping with literature data 
TABLE 1 | Values of demographic indices (1a), psychometric measures (1b), and sleep parameters (1c) of NT1 patients and sc-EDS subjects with NREM naps.

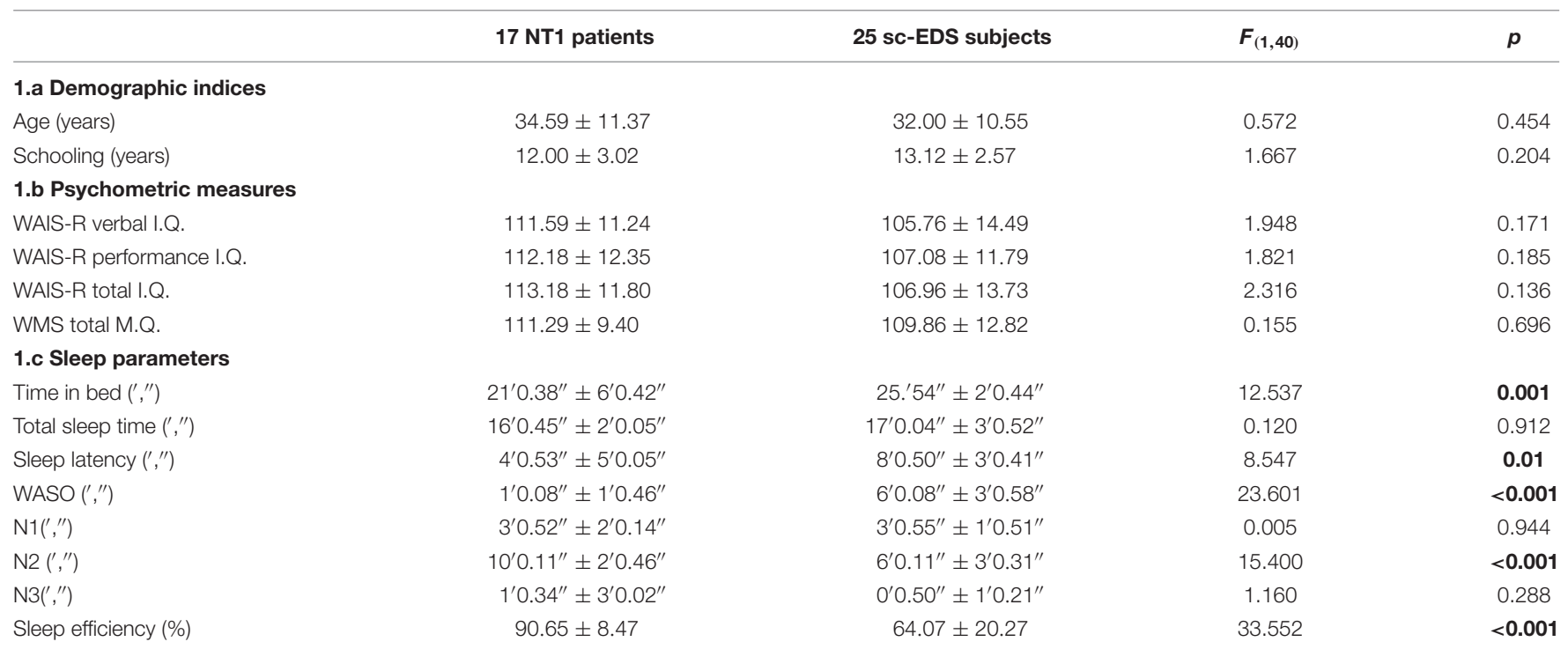

NT1, narcolepsy type 1; I.Q., intelligence quotient; M.Q., memory quotient; WAIS-R, Wechsler Adult Intelligence Scale Revised; WMS, Wechsler Memory Scale; ', minutes;" seconds; N1, N2, N3, stage 1, 2, 3 of NREM sleep ; $p$ values in bold are statistically significant.

TABLE 2 | Occurrences of dreams, white dreams, and no dreams in NREM and SOREM naps of NT1 patients and sc-EDS subjects.

\begin{tabular}{|c|c|c|c|c|c|c|c|c|c|}
\hline 17 NT1 patients & \multicolumn{4}{|c|}{ NREM naps } & \multicolumn{4}{|c|}{ SOREMP naps } & No sleep \\
\hline Trial 1 & 2 & 0 & 0 & 2 & 14 & 11 & 2 & 1 & 1 \\
\hline Trial 2 & 6 & 1 & 3 & 2 & 11 & 7 & 3 & 1 & \\
\hline Trial 3 & 6 & 3 & 3 & 0 & 11 & 6 & 4 & 1 & \\
\hline Trial 4 & 6 & 2 & 2 & 2 & 10 & 7 & 1 & 2 & 1 \\
\hline \multicolumn{10}{|c|}{25 sc-EDS subjects } \\
\hline Trial 1 & 20 & 16 & 1 & 3 & 2 & 2 & 0 & 0 & 3 \\
\hline Trial 2 & 23 & 6 & 6 & 11 & 1 & 0 & 1 & 0 & 1 \\
\hline Trial 3 & 24 & 11 & 3 & 10 & 0 & 0 & 0 & 0 & 1 \\
\hline Trial 4 & 18 & 6 & 5 & 7 & 2 & 0 & 1 & 1 & 5 \\
\hline
\end{tabular}

T, total number of naps; $D$, dream; WD, white dream; ND, no dream.

(Pizza et al., 2013). Indeed, both the proportion of failure to take a nap and the frequency of spontaneous awakening (i.e., before the end of trial) in NREM naps were significantly lower (while sleep efficiency was higher) in NT1 patients compared with scEDS subjects. Moreover, NREM naps of NT1 patients had not only a shorter sleep latency and a lower proportion of WASO but also a longer duration of $\mathrm{N} 2$ sleep (usually until the end of trial: see Table 3) compared with naps of sc-EDS subjects, which were concluded often in other NREM sleep stages or wakefulness.

\section{Dream Data}

The values of the indicators of recall and structural organization of NREM-DEs converge to indicate that the low frequency of NREM-DEs reported by NT1 patients depends on a failure in recall after awakening rather than in generation during sleep. Indeed, while the frequency of white dreams was significantly higher in NREM naps of NT1 patients (42.86\%) compared with both NREM naps of sc-EDS subjects (17.48\%) and SOREMP naps of NT1 patients themselves (20\%), the frequency of all NREM-DEs (i.e., dreams plus white dreams) was similar in the two groups (67.86 and $61.17 \%$, respectively). Notably, this frequency was comparable also with those of DEs reported by healthy subjects after both daytime NREM (around 60\%: for review, see Nielsen, 2011b) and nighttime NREM sleep (Pivik and Foulkes, 1968).

Moreover, the dream findings, if considered together with the PSG ones, went against the two main predictions of the arousal-retrieval model. First, the higher duration of WASO 
TABLE 3 | Sleep stage or wakefulness at the end of MSLT trials resulting in NREM naps with dream, white dream, or no dream.

\begin{tabular}{|c|c|c|c|c|c|c|c|c|c|c|c|c|c|c|c|c|c|c|c|}
\hline \multirow[t]{2}{*}{17 NT1 patients } & \multicolumn{3}{|c|}{ NAP 1} & \multicolumn{3}{|c|}{ NAP 2} & \multicolumn{3}{|c|}{ NAP 3} & \multicolumn{3}{|c|}{ NAP 4} & \multicolumn{3}{|c|}{ NAP 5} & \multicolumn{4}{|c|}{ Total } \\
\hline & D & WD & ND & D & WD & ND & D & WD & ND & D & WD & ND & D & WD & ND & $\mathbf{T}$ & D & WD & ND \\
\hline N1 & 0 & 0 & 0 & 0 & 0 & 0 & 0 & 1 & 0 & 0 & 0 & 0 & 0 & 0 & 0 & 1 & 0 & 1 & 0 \\
\hline $\mathrm{N} 2$ & 0 & 0 & 2 & 1 & 2 & 1 & 2 & 2 & 0 & 2 & 2 & 2 & 0 & 1 & 1 & 18 & 5 & 7 & 6 \\
\hline N3 & 0 & 0 & 0 & 0 & 1 & 0 & 1 & 0 & 0 & 0 & 1 & 0 & 1 & 2 & 1 & 7 & 2 & 4 & 1 \\
\hline Wakefulness & 0 & 0 & 0 & 0 & 0 & 1 & 0 & 0 & 0 & 0 & 0 & 0 & 0 & 0 & 1 & 2 & 0 & 0 & 2 \\
\hline Total & 0 & 0 & 2 & 1 & 3 & 2 & 3 & 3 & 0 & 2 & 3 & 2 & 1 & 3 & 3 & 28 & 7 & 12 & 9 \\
\hline \multicolumn{20}{|c|}{25 sc-EDS subjects } \\
\hline N1 & 4 & 0 & 0 & 0 & 1 & 0 & 3 & 0 & 0 & 1 & 0 & 1 & 0 & 0 & 2 & 12 & 8 & 1 & 3 \\
\hline N2 & 6 & 1 & 2 & 2 & 4 & 4 & 3 & 2 & 4 & 3 & 2 & 2 & 4 & 0 & 2 & 41 & 18 & 9 & 14 \\
\hline N3 & 0 & 0 & 0 & 1 & 1 & 1 & 2 & 1 & 3 & 1 & 2 & 2 & 0 & 2 & 2 & 18 & 4 & 6 & 8 \\
\hline Wakefulness & 6 & 0 & 1 & 3 & 0 & 6 & 3 & 0 & 3 & 1 & 1 & 2 & 2 & 1 & 3 & 32 & 15 & 2 & 15 \\
\hline Total & 16 & 1 & 3 & 6 & 6 & 11 & 11 & 3 & 10 & 6 & 5 & 7 & 6 & 3 & 9 & 103 & 45 & 18 & 40 \\
\hline
\end{tabular}

T, total number of naps; $D$, dream; WD, white dream; ND, no dream.

after sleep onset should lead to expectation of a higher DE recall frequency (Koulack and Goodenough, 1976; Vallat et al., 2017) in sc-EDS subjects compared with NT1 patients (in whom the value of WASO was negligible: $6^{\prime} 0.08^{\prime \prime} \pm 3^{\prime} 0.58^{\prime \prime}$ vs $1^{\prime} 0.08^{\prime \prime} \pm 1^{\prime} 0.46^{\prime \prime}$, respectively, see Table 1 ), contrary to the findings obtained. Second, the NREM naps of NT1 patients were concluded prevalently by a provoked (i.e., abrupt) awakening, which should facilitate dream recall (Goodenough et al., 1965) and, thus, lead to a higher proportion of dreams compared with white dreams in NT1 patients than sc-EDS subjects, again contrary to our findings.

Finally, also the values of the indicators of length and structural organization of NREM-DEs (lower than those of SOREM-DEs of NT1 patients but higher than those of NREMDEs of sc-EDS subjects: see Table 4) were coherent with the hypothesis of a recall failure, under the assumption that recall is easier for more complex and long DEs (Foulkes and Schmidt, 1983). Indeed, NT1 patients are used to retrieve a wealth of vivid and bizarre DE contents after daytime SOREMP as well as after nighttime REM sleep (Broughton, 1982; Fosse, 2000). Notably, as shown by studies on healthy subjects, both vividness (Salzarulo and Cipolli, 1979; Foulkes and Schmidt, 1983) and bizarreness of DE contents (Cipolli et al., 1993; Nielsen, 2011a) facilitate their retrieval after awakening These features of DE contents clearly concur to determine the positive relationship between the quality of DE contents (according to the definition of this notion proposed by Fazekas et al., 2019) and the effectiveness of recall. The specific role played by these features in the general process of DE recall might be established in studies carried out on participants undergone some preliminary training (see below).

Explaining the failure in recall (i.e., the discrepancy between the occurrence and reporting) of NREM-DE in terms of a negative attitude of NT1 patients toward recall of short and poorly structured DEs appears plausible also on the basis of the historical evolution of the estimates of DE frequency. Indeed, the proportions of DEs recalled have been enhanced over years from the early estimates (Dement and Kleitman, 1957a,b) to the normative ones (see Nielsen, 2000) by interviewing participants (a) using a more extensive definition of the notion of "dream" (including any mental experience during sleep), (b) after a preliminary session for adaptation to laboratory content and task of dream recall (for review, see Cohen, 1979), and (c) using articulated questions to guide participants in retrieving features of DE relevant for the study aim and design (Kahn et al., 2000). It is apparent that first-diagnosed NT1 patients interviewed after MSLT naps had not any previous experience of dream reporting in laboratory context. It seems thus plausible that they are likely to desist from attempting to retrieve the less vivid and bizarre contents as usually the NREM-sleep ones are compared with those they are used to recall effortlessly after awakening from (SO)REM sleep (Broughton, 1982; Fosse, 2000).

Also, the findings of two recent studies speak in favor of this explanation. The former study showed that a low frequency of dream recall (25.64\%) is obtained by administering a dream questionnaire after NT1 patients got up on their own (Schredl and Olbrich, 2019). This low recall frequency (albeit not distinguishing NREM-DEs from SOREMP-DEs) confirms that untrained NT1 patients estimate to have had a DE when some contents are easily accessible on delayed (after some hours) as well as on immediate recall (as in the present study). The latter study, carried out using a within-subject design and estimating DE recall of NT patients in terms of subjective evaluation (yes/no), showed that a lower delta power over parietal and centro-parietal areas is predictive of successful (i.e., yes) recall for DE of both NREM and REM naps (D'Atri et al., 2019). The large overlapping of the EEG correlates of successful recall after SOREMP and NREM naps suggests that REM and NREM sleep share a similar machinery for dream recall in NT1 patients as well as in healthy subjects (Siclari et al., 2017). This finding, in weakening the alternative hypothesis of an intrinsically lower capacity of NREM sleep of NT1 patients for memory encoding of DE contents, further corroborates the interpretative hypothesis put forward here that a negative attitude toward recall is responsible of the lower frequency of NREM-DEs reported compared the generated ones. 


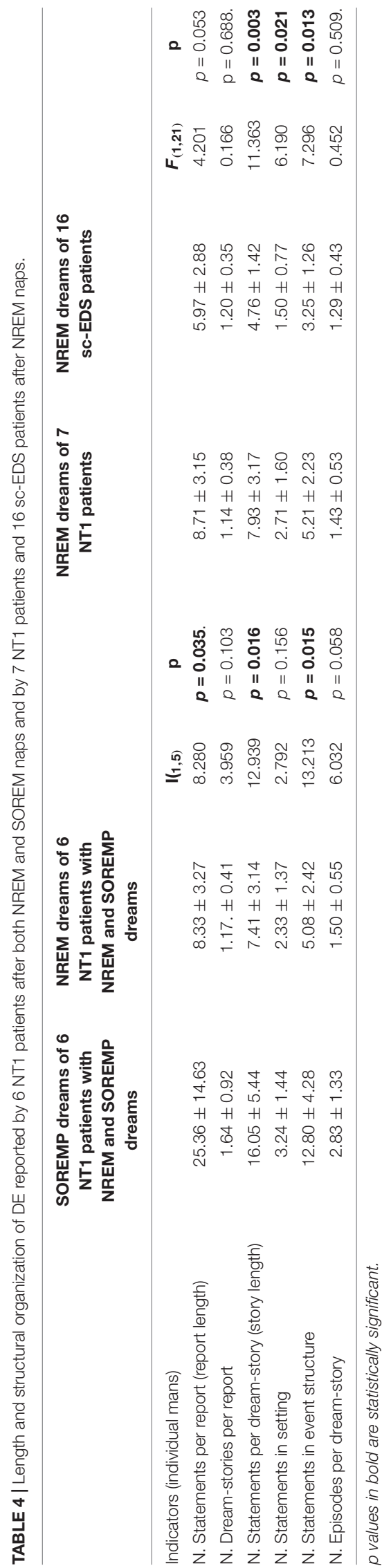

Notably, also the residual difference (about 15\%) between the present estimate of the "real" NREM-DE frequency (as sum of dreams and white dreams) and that of Vogel's (1976) experimental study (i.e., with planned awakening and immediate DE recall) on NT1 patients may be accounted for in keeping with the above hypothesis. Indeed, Vogel's patients not only were under a greater sleep pressure at the moment of one or two around-noon naps compared with patients undergone MSLT trial every, $2 \mathrm{~h}$ but also underwent a preliminary training for DE recall. As shown by studies on healthy subjects, a great sleep pressure enhances such dreamlike features as vividness and bizarreness of DE contents after daytime (Carr and Nielsen, 2015) as well as nighttime NREM sleep (Nielsen et al., 2005), and a preliminary training enhances the rate of successful recall of specific features of dream content (Kahn et al., 2000).

It appears thus plausible that some preliminary training during the diagnostic procedure (for example, after the first night of PSG recordings), by modifying the negative attitude toward recall of less vivid and bizarre DE contents in NT1 patients undergone MSLT, can enhance the proportion of DEs reported (i.e., dreams) after NREM as well as SOREMP naps (their proportions of white dreams in the present study being 42.86 and 20\%, respectively, see Table 2 ). Moreover, the D'Atri et al. (2019) study showed a large overlapping of EEG correlates of successful recall after SOREMP and NREM naps of NT1 patients. Therefore, gathering this item of evidence in future studies would concur to confirm that MSLT may be used as a parsimonious (being within the clinical routine) and extended (from mid-morning to late afternoon) multiple-nap protocol to investigate the characteristics of content and structural organization of NREMDEs as well as of SOREMP-DEs (Cipolli et al., 2020). Obviously MSLT studies, given the intrinsic limitation of the fixed nap duration, cannot substitute completely the experimental ones but may provide useful insights into the general process of DE generation.

\section{Future Directions}

Collecting an amount of DE reports fairly comparable to that of DEs generated by NT1 patients during NREM sleep (namely, in about two thirds of NREM naps) would allow not only to identify (by using spectral analysis techniques) the global and local EEG correlates of DE recall but also cast light on $\mathrm{DE}$ generation as a continuum across sleep stages. This issue has been recently pointed out (Nielsen, 2017) as at least crucial as that of the wake-sleep continuity (Schredl and Hofmann, 2003) for the understanding of the relationships between the neurophysiological EEG signals and the functioning of the cognitive processes underlying $\mathrm{DE}$ generation. As shown by studies on healthy subjects, the contents of DEs result from the often repeated activation of more or less recent memories and some rebinding of their features in subsequent sleep stages. This iterative processing is indicated by both the interrelated (i.e., similar) contents present in NREM- and REM-DEs of the same night (Rechtschaffen et al., 1963b; Cipolli et al., 1988) and the repeated incorporation of pre-sleep stimuli as contents into DEs of subsequent sleep stages (Wamsley et al., 2010). 
Moreover, the prevalent but not exclusive transition from N1 to SOREMP sleep (Drakatos et al., 2013a; Cipolli et al., 2020) can offer the opportunity to observe two potentially different types of across-stage continuity by comparing the content and structural organization of DEs reported after MSLT naps in which SOREMP has been preceded by only N1 or also N2 sleep. Since frontal theta activity increases from the first to the last $30 \mathrm{~s}$ of N1 (as shown in healthy subjects: Picchioni et al., 2008), this comparison would clarify whether the complexity of the story-like plot of SOREMPDEs is facilitated by the continuity with the high imagery typical of N1 (i.e., the construction of a mental fictive scenario, with spatial location of the imagined event: Hori et al., 1994; Stenstrom et al., 2012) or by the spreading of associative networks typical of N2 (as indicated by spindle density: Studte et al., 2015; Sopp et al., 2018).

These indications might be complemented by those gathered using properly experimental protocols (to be applied on voluntary NT1 patients after diagnostic routine) adequate to establish how pre-sleep stimuli or new tasks are incorporated as contents into the DEs of subsequent naps with only N1, N1 plus N2 sleep, or also SOREMP sleep. The variations in the rate and modality of incorporations according to the stage of prior sleep may shed new light on the role played in particular by $\mathrm{N} 1$ in the process of sleep-dependent memory consolidation not only per se (as already shown on healthy subjects: Lahl et al., 2008; Wamsley et al., 2010), but also in interaction with SOREMP sleep (which is only observable on NT1 patients).

\section{CONCLUSION}

This study ascertained that the frequency of DEs generated during NREM naps of NT1 patients at MSLT is higher than usually estimated and fully comparable to those both of scEDS subjects and (even more importantly) of healthy subjects. Moreover, the structural organization of the reported NREMDEs indicated that the discrepancy between the frequencies of DEs generated and reported depends on the attitude toward recall of NT1 patients and, thus, can be reversed by some training before MSLT trials. Given the peculiar neurophysiology of sleep in NT1 patients, enhancing the frequency and accuracy of their DE reports after NREM naps may provide new insights into some

\section{REFERENCES}

American Academy of Sleep Medicine [AASM] (2014). International Classification of Sleep Disorders (Third Edition). Darien, IL: American Academy of Sleep Medicine.

Bassetti, C. L. A., Adamantidis, A., Burdakov, D., Han, F., Gay, S., Kallweit, U., et al. (2019). Narcolepsy-clinical spectrum, aetiopathophysiology, diagnosis and treatment. Nat. Rev. Neurol. 15, 519-539. doi: 10.1038/s41582-019-0226-229

Bellucci, C., Vandi, S., Iloti, M., Pizza, F., Russo, P. M., Tuozzi, G., et al. (2016). Dissociated rapid eye movement sleep dream experiences in type 1 narcolepsy: a case report. Sleep Med. 19, 150-152. doi: 10.1016/j.sleep.2015.07.036

Benbadis, S. R., Wolgamuth, B. R., Perry, M. C., and Dinner, D. S. (1995). Dreams and rapid eye movement sleep in the multiple sleep latency test. Sleep 18, 105-108. doi: 10.1093/sleep/18.2.105

Broughton, R. J. (1982). Neurology and dreaming. Psychiat. J. Univ. Ottawa 7, $101-110$. issues (such as the across-stages continuity in the functioning of the cognitive processes underlying DE generation) the approach to which is harder in healthy subjects.

\section{DATA AVAILABILITY STATEMENT}

The raw data supporting the conclusions of this article will be made available by the authors, without undue reservation.

\section{ETHICS STATEMENT}

The studies involving human participants were reviewed and approved by the Ethics Committee of the University of Bologna. The participants provided their written informed consent to participate in this study.

\section{AUTHOR CONTRIBUTIONS}

CC, FP, MM, and GP conceived and designed the study. CB collected the dream data. GT and SV collected polysomnographic data. MM and CB carried outs statistical analysis. CC and FP wrote the first draft. All the authors approved the final version of the manuscript after the changes they suggested.

\section{FUNDING}

This study was supported in part by funds of the University of Bologna for fundamental research to MM and GP in 2018-20 (RFO_MAZZETTI 2018, 2019, RFO_PLAZZI 2018, 2019).

\section{ACKNOWLEDGMENTS}

We are indebted to M. Iloti, G. Neccia, and A. Maltoni for technical assistance in sleep recordings, to L. Vignatelli for visual scoring of sleep recordings, to M. Baggio and E. Rigotti for linguistic analysis of dream reports, and to C. Baroncini for linguistic assistance in the preparation of the manuscript.

Carr, M., and Nielsen, T. (2015). Daydreams and nap dreams: Content comparisons. Conscious. Cogn. 36, 196-205. doi: 10.1016/j.concog.2015. 06.012

Carskadon, M. A., Dement, W. C., Mitler, M. M., Roth, T., Westbrook, P. R., and Keenan, S. (1986). Guidelines for the multiple sleep latency test (MSLT): a standard measure of sleepiness. Sleep 9, 519-524. doi: 10.1093/sleep/9.4.519

Cipolli, C., and Poli, D. (1992). Story structure in verbal reports of mental sleep experience after awakening in REM sleep. Sleep 15, 133-142. doi: 10.1093/sleep/ 15.2.133

Cipolli, C., Bellucci, C., Mattarozzi, K., Mazzetti, M., Tuozzi, G., and Plazzi, G. (2008). Story-like organization of REM-dreams in patients with narcolepsycataplexy. Brain Res. Bull. 77, 206-213. doi: 10.1016/j.brainresbull.2008.07. 012

Cipolli, C., Bolzani, R., and Tuozzi, G. (1998). Story-like organization of dream experience in different periods of REM sleep. J. Sleep Res. 7, 13-19. doi: 10.1046/ j.1365-2869.1998.00094 
Cipolli, C., Bolzani, R., Cornoldi, C., De Beni, R., and Fagioli, I. (1993). Bizarreness Effect in Dream Recall. Sleep 16, 163-170. doi: 10.1093/sleep/16.2.163

Cipolli, C., Fagioli, I., Baroncini, P., Fumai, A., Marchiò, B., and Sancini, M. (1988). The thematic continuity of mental experiences in REM and NREM sleep. Int. J. Psychophysiol. 6, 307-313. doi: 10.1016/0167-8760(88)90018-90019

Cipolli, C., Pizza, F., Bellucci, C., Mazzetti, M., Tuozzi, G., Vandi, S., et al. (2020). Structural organization of dream experience in SOREMP sleep of patients with narcolepsy type 1. Sleep 43:zsaa012. doi: 10.1093/ sleep/zsaa012

Cohen, D. B. (1972). Failure to recall dream content: contentless vs dreamless reports. Percept. Mot. Skills 34, 1000-1002. doi: 10.2466/pms.1972.34.3.1000

Cohen, D. B. (1979). Sleep And Dreaming: Origins, Nature And Functions. Oxford, UK: Pergamon Press.

D’Atri, A., Scarpelli, S., Schiappa, C., Pizza, F., Vandi, S., Ferrara, M., et al. (2019). Cortical activation during sleep predicts dream experience in narcolepsy. Ann. Clin. Transl. Neurol. 6, 445-455. doi: 10.1002/acn3.718

Dement, W., and Kleitman, N. (1957a). Cyclic variations in EEG during sleep and their relation to eye movements, body motility, and dreaming. Electroencephalogr. Clin. Neurophysiol. 9, 673-690. doi: 10.1016/0013-4694(57) 90088-90083

Dement, W., and Kleitman, N. (1957b). The relation of eye movements during sleep to dream activity: an objective method for the study of dreaming. J. Exp. Psychol. 53, 339-346. doi: 10.1037/h0048189

Dement, W., Rechtschaffen, A., and Gulevich, G. (1966). The nature of the narcoleptic sleep attack. Neurology 16, 18-33. doi: 10.1212/wnl. 16.1.18

Dodet, P., Chavez, M., Leu-Semenescu, S., Golmard, J. L., Arnulf, I., et al. (2015). Lucid dreaming in narcolepsy. Sleep 8, 487-497. doi: 10.5665/sleep.4516

Drakatos, P., Kosky, C. A., Higgins, S. E., Muza, R. T., Williams, A. J., and Leschziner, G. D. (2013a). First rapid eye movement sleep periods and sleeponset rapid eye movement periods in sleep-stage sequencing of hypersomnias. Sleep Med. 14, 897-901. doi: 10.1016/j.sleep.2013.03.021

Drakatos, P., Suri, A., Higgins, S. E., Ebrahim, I. O., Muza, R. T., Kosky, C. A., et al. (2013b). Sleep stage sequence analysis of sleep onset REM periods in the hypersomnias. J. Neurol. Neurosurg. Psychiatry 84, 223-227. doi: 10.1136/jnnp2012-303578

Fazekas, P., Nemeth, G., and Overgaard, M. (2019). White dreams are made of colours: What studying contentless dreams can teach about the neural basis of dreaming and conscious experiences. Sleep Med. Rev. 43, 84-91. doi: 10.1016/j. smrv.2018.10.005

Fosse, R. (2000). REM mentation in narcoleptics and normals: an empirical test of two neurocognitive theories. Conscious. Cogn. 9, 488-509. doi: 10.1006/ccog. 2000.0466

Foiulkes, D. (1962). Dream reports from different stages of sleep. J. Abn. Soc. Psychol. 65, 14-25. doi: 10.1037/h0040431

Foulkes, D., and Schmidt, M. (1983). Temporal sequence and unit composition in dream reports from different stages of sleep. Sleep 6, 265-280. doi: 10.1093/ sleep/6.3.265

Goodenough, D. R., Lewis, H. B., Shapiro, A., Jaret, L., and Sleser, I. (1965). Dream reporting following abrupt and gradual awakenings from different types of sleep. J. Pers. Soc. Psychol. 2, 170-179. doi: 10.1097/00006842-196507000196507005

Hori, T., Hayashi, M., and Morikawa, T. (1994). “Topographical EEG changes and hypnagogic experience," in Sleep Onset: Normal and Abnormal Processes, eds R. D. Ogilvie and J. R. Harsh (Wahshington, DC: American Psycholological Association), 237-253.

Iber, C., Ancoli-Isarel, S., Chesson, A. L., and Quan, S. F. (2007). The Aasm Manual for the Scoring of Sleep and Associated Events: Rules, Terminology, and Technical Specifications 1st Ed, Darien, IL: American Academy of Sleep Medicine.

Kahn, D., Stickgold, R., Pace-Schott, E. F., and Hobson, J. A. (2000). Dreaming and waking consciousness: a character recognition study. J. Sleep Res. 9, 317-325. doi: 10.1046/j.1365-2869.2000.00213.x

Koulack, D., and Goodenough, D. (1976). Dream recall and dream recall failure: an arousal-retrieval model. Psychol. Bull. 83, 975-984. doi: 10.1037/0033-2909. 83.5.975

Lahl, O., Wispel, C., Willigens, B., and Pietrowsky, R. (2008). An ultra short episode of sleep is sufficient to promote declarative memory performance. J. Sleep Res. 17, 3-10. doi: 10.1111/j.1365-2869.2008.00622
Littner, M. R., Kushida, C., Wise, M., Davila, D. G., Morgenthaler, T., Lee-Chiong, T., et al. (2005). Practice parameters for clinical use of the multiple sleep latency test and the maintenance of wakefulness test. Sleep 28, 113-121. doi: 10.1093/ sleep/28.1.113

Mandler, J., and Johnson, N. S. (1977). Remembrance of things parsed: story structure and recall. Cogn. Psychol. 9, 111-151. doi: 10.1016/0010-0285(77) 90006-90008

Mazzetti, M., Bellucci, C., Mattarozzi, K., Plazzi, G., Tuozzi, G., and Cipolli, C. (2010). REM-dreams recall in patients with narcolepsy-cataplexy. Brain Res. Bull. 81, 133-140. doi: 10.1016/j.brainresbull.2009.10.021

Mignot, E., Lin, L., Finn, L., Lopes, C., Pluff, K., Sundstrom, M. L., et al. (2006). Correlates of sleep-onset REM periods during the Multiple Sleep Latency Test in community adults. Brain 129, 1609-1623. doi: 10.1093/brain/awl079

Nielsen, T. (2000). A review of mentation in REM and NREM sleep: "covert" REM sleep as a possible reconciliation of two opposing models. Behav. Brain Sci. 23, 851-866. doi: 10.1017/s0140525x0000399x

Nielsen, T. (2011a). "Dream analysis and classification: the reality simulation perspective," in Principles and Practice of Sleep Medicine, 5th Edn, eds M. Kryger, T. Roth, and W. C. Dement (New York: Elsevier), 595-603.

Nielsen, T. (2011b). "Ultradian, circadian, and sleep-dependent features of dreaming," in Principles and Practice of Sleep Medicine, 5th Edn, eds M. Kryger, T. Roth, and W. C. Dement (New York: Elsevier), 576-584.

Nielsen, T. (2017). Microdream neurophenomenology. Neurosci. Consc. 1:nix001. doi: 10.1093/nc/nix001

Nielsen, T., Stenstrom, P., Takeuchi, T., Saucier, S., Lara-Carrasco, J., Solomonova, E., et al. (2005). Partial REM-sleep deprivation increases the dreamlike quality of mentation from REM sleep and sleep onset. Sleep 28, 1083-1089. doi: 10. 1093/sleep/28.9.1083

Picchioni, D., Fukunaga, M., Carr, W. S., Braun, A. R., Balkin, T. J., Duyn, J. H., et al. (2008). fMRI differences between early and late stage-1 sleep. Neurosci. Lett. 441, 81-85. doi: 10.1016/j.neulet.2008.06.010

Pivik, T., and Foulkes, D. (1968). NREM mentation: relation to personality, orientation time, and time of night. J. Consult. Clin. Psychol. 32, 144-151. doi: $10.1037 /$ h0025489

Pizza, F., Moghadam, K. K., Vandi, S., Detto, S., Poli, F., Mignot, E., et al. (2013). Daytime continuous polysomnography predicts MSLT results in hypersomnias of central origin. J. Sleep Res. 22, 32-40. doi: 10.1111/j.1365-2869.2012.01032.x

Rak, M., Beitinger, P., Steiger, A., Schredl, M., and Dresler, M. (2015). Increased lucid dreaming frequency in narcolepsy. Sleep 38, 787-792. doi: 10.5665/sleep. 4676

Rechtschaffen, A., Vogel, G., and Shaikun, G. (1963a). Interrelatedness of mental activity during sleep. Arch. Gen. Psychiatry 9, 536-547. doi: 10.1001/archpsyc. 1963.01720180008002

Rechtschaffen, A., Wolpert, E. A., Dement, W. C., Mitchell, S. A., and Fisher, C. (1963b). Nocturnal sleep of narcoleptics. Electroencephalogr. Clin. Neurophysiol. 15, 599-609. doi: 10.1016/0013-4694(63)90032-90034

Richardson, G. S., Carskadon, M. A., Flagg, W., Van den Hoed, J., Dement, W. C., and Mitler, M. M. (1978). Excessive daytime sleepiness in man: multiple sleep latency measurement in narcoleptic and control subjects. Electroencephalogr. Clin. Neurophysiol. 45, 621-627. doi: 10.1016/0013-4694(78)90162-90161

Salzarulo, P., and Cipolli, C. (1979). Linguistic organization and cognitive implications of REM and NREM sleep-related reports. Percept. Mot. Skills 49, 767-777. doi: 10.2466/pms.1979.49.3.767

Schinkelshoek, M., de Wit, K., Bruggink, V., Fronczek, R., and Lammers, G. J. (2018). Sleep-state and dream per-ception in sleep disorders. J. Sleep Res. 27:296.

Schredl, M. (2009). Dreams in patients with sleep disorders. Sleep Med. Rev. 13, 215-221. doi: 10.1016/j.smrv.2008.06.002

Schredl, M., and Hofmann, F. (2003). Continuity between waking activities and dream activities. Consc. Cogn. 12, 298-308. doi: 10.1016/s1053-8100(02)0007277

Schredl, M., and Olbrich, K. I. (2019). Dream recall after Multiple Sleep Latency Test naps with and without REM sleep. Int. J. Dream Res. 12, 81-84.

Siclari, F., Baird, B., Perogamvros, L., Bernardi, G., LaRocque, J. J., Riedner, B., et al. (2017). The neural correlates of dreaming. Nat. Neurosci. 20, 872-878. doi: $10.1038 /$ nn. 4545.31

Sopp, M. R., Michael, T., and Mecklinger, A. (2018). Effects of early morning nap sleep on associative memory for neutral and emotional stimuli. Brain Res. 1698, 29-42. doi: 10.1016/j.brainres.2018.06.020 
Stenstrom, P., Fox, K., Solomonova, E., and Nielsen, T. (2012). Mentation during sleep onset theta bursts in a trained participant: A role for NREM stage 1 sleep in memory processing? Int. J. Dream Res. 5, 37-46.

Studte, S., Bridger, E., and Mecklinger, A. (2015). Nap sleep preserves associative but not item memory performance. Neurobiol. Learn. Mem. 120, 84-93. doi: 10.1016/j.nlm.2015.02.012

Vallat, R., Lajnef, T., Eichenlaub, J. B., Berthomier, C., Jerbi, K., Morlet, D., et al. (2017). Increased Evoked Potentials to Arousing Auditory Stimuli during Sleep: Implication for the Understanding of Dream Recall. Front. Hum. Neurosci. 11:132. doi: 10.3389/fnhum.2017.0013

Vandi, S., Pizza, F., Antelmi, E., Neccia, G., Iloti, M., Mazzoni, A., et al. (2019). A standardized test to document cataplexy. Sleep Med. 53, 197-204. doi: 10.1016/ j.sleep.2017.08.021

Vignatelli, L., Plazzi, G., Barbato, A., Ferini-Strambi, L., Manni, R., Pompei, F., et al. (2003). Italian version of the Epworth sleepiness scale: external validity. Neurol. Sci 23, 295-300. doi: 10.1007/s100720300004

Vogel, G. (1976). "Mentation reported from naps of narcoleptics," in Narcolepsy, eds C. Guilleminault, W. Dement, and P. Passouant (New York: Spectrum), 161-168.

Waihrich, E. S., Rodrigues, R. N., Silveira, H. A., Fróes Fda, F., and Rocha, G. H. (2006). Comparative analysis of multiple sleep latency tests (MSLT) parameters and occurrence of dreaming in patients with daytime sleepiness of narcoleptic and non-narcoleptic origin. Arq. Neuropsiquiatry 64, 958-962. doi: 10.1590/ s0004-282x2006000600014

Wamsley, E. J., Perry, K., Djonlagic, I., Reaven, L. B., and Stickgold, R. (2010). Cognitive replay of visuomotor learning at sleep onset: temporal dynamics and relationship to task performance. Sleep 33, 59-68. doi: 10.1093/sleep/ 33.1.59

Wechsler, D. (1981). Wechsler adult intelligence scale-Manual revised. Cleveland: The Psychological Corporation.

Wechsler, D. (1987). Wechsler Memory Scale-Revised Manual. New York: The Psychological Corporation.

Conflict of Interest: The authors declare that the research was conducted in the absence of any commercial or financial relationships that could be construed as a potential conflict of interest.

Copyright (c) 2020 Cipolli, Pizza, Bellucci, Mazzetti, Tuozzi, Vandi and Plazzi. This is an open-access article distributed under the terms of the Creative Commons Attribution License (CC BY). The use, distribution or reproduction in other forums is permitted, provided the original author(s) and the copyright owner(s) are credited and that the original publication in this journal is cited, in accordance with accepted academic practice. No use, distribution or reproduction is permitted which does not comply with these terms. 